\title{
Prediction of Geomagnetic Storm Strength from Inner Heliospheric In Situ Observations
}

\author{
M. Kubicka ${ }^{1}$, C. Möstl ${ }^{1,2}$, T. Amerstorfer ${ }^{1}$, P. D. Boakes ${ }^{1,2}$, L. Feng ${ }^{3}$, J. P. Eastwood ${ }^{4}$, O. \\ Törmänen ${ }^{1,5}$ \\ ${ }^{1}$ Space Research Institute, Austrian Academy of Sciences, 8042 Graz, Austria \\ ${ }^{2}$ Kanzelhöhe Observatory-IGAM, Institute of Physics, University of Graz, 8010 Graz, \\ Austria \\ ${ }^{3}$ Purple Mountain Observatory, Chinese Academy of Sciences. West Beijing Road 2 \\ Nanjing, 210008, China \\ ${ }^{4}$ Space and Atmospheric Physics, The Blackett Laboratory, Imperial College London, \\ London $S W^{r}$ 2AZ, UK \\ ${ }^{5}$ Aalto University, School of Electrical Engineering, 02150 Espoo, Finland \\ christian.moestl@oeaw.ac.at
}

\begin{abstract}
Prediction of the effects of coronal mass ejections (CMEs) on Earth strongly depends on knowledge of the interplanetary magnetic field southward component, $B_{z}$. Predicting the strength and duration of $B_{z}$ inside a CME with sufficient accuracy is currently impossible, which forms the so-called $B_{z}$ problem. Here, we provide a proof-of-concept of a new method for predicting the CME arrival time, speed, $B_{z}$ and the resulting $D s t$ index at Earth based only on magnetic field data, measured in situ in the inner heliosphere ( $<1$ AU). On 2012 June 12-16, three approximately Earthward-directed and interacting CMEs were observed the by the STEREO imagers, and by Venus Express (VEX) in situ at 0.72 AU, 6 degree away from the Sun Earth line. The CME kinematics are calculated using the drag-based and WSA-Enlil models, constrained by the arrival time at VEX, resulting in the CME arrival time and speed at Earth. The CME magnetic field strength is scaled with a power law from $V E X$ to Wind. Our investigation shows promising results for the Dst forecast (predicted: -96 and $-114 \mathrm{nT}$ (from 2 Dst models), observed: $-71 \mathrm{nT}$ ), for the arrival speed (predicted: $531 \pm 23 \mathrm{~km} \mathrm{~s}^{-1}$, observed: $488 \pm 30 \mathrm{~km} \mathrm{~s}^{-1}$ ) and timing (6士1 hours after actual arrival time). The prediction lead time is 21 hours. The method may be applied to vector magnetic
\end{abstract}


field data from a spacecraft at an artificial Lagrange point between the Sun and Earth, or to data taken by any spacecraft temporarily crossing the Sun-Earth line.

Subject headings: solar-terrestrial relations — solar wind — Sun: coronal mass ejections (CMEs) — Sun: heliosphere

\section{Introduction}

Extreme space weather events are mostly driven by coronal mass ejections (CMEs) and their interplanetary manifestations, called interplanetary CMEs or ICMEs (e.g. Zhang et al. 2007). They have potential to be hazardous for an extensive list of different technologies, therefore the precise forecasting of such space weather events becomes increasingly important. Many different methods exist to predict the initial speeds, directions and shapes of CMEs and the evolution of CMEs through the inner heliosphere, but none of them are able to accurately predict the magnetic field components, in particular the southward $B_{z}$ component, which is crucial to calculate a CME's geomagnetic effectiveness (e.g. Dungev 1961; Rostoker \& Fälthammar 1967; Burton et al. 1975; Hoeksema \& Zhao 1992; O'Brien \& McPherron 2000).

Here, we present a proof-of-concept for predicting the strength of geomagnetic storms caused by CMEs, using in situ data from a spacecraft situated in the inner heliosphere, i.e. in the space $<1$ AU, and close to the Sun-Earth line. By definition (Rouillard et al. 2011) we use the term ICME (interplanetary CME) for the in situ observation of a CME. With measurements from Venus Express (VEX), located at 0.72 AU, our method predicts the ICME arrival speed and time, the magnetic field of the ICME and the resulting disturbance storm time (Dst) index at Earth. Lindsay et al. (1999) provided pioneering work on the predictability of the Dst index with the help of a solar wind monitor located inside 1 AU. We extend this work by introducing a completely new methodology for this type of prediction. So far, typical CME forecasts show an error in arrival time of 6 to 18 hours (e.g. Colaninno et al. 2013; Möstl et al. 2014; Vršnak et al. 2014; Mays et al. 2015; Rollett et al. 2016) and cannot provide the magnetic field, or use very general models and relationships to find an approximate Dst forecast (Tobiska et al. 2013; Savani et al. 2015). Predicting the $D s t$ index from data acquired at the Sun-Earth L1 point provides high accuracy but has the disadvantage of a very short prediction lead time of only 30 to 60 minutes (e.g. Bala \& Reiff 2012). We apply our method to a period where VEX was near the Sun-Earth line that might include contributions from three potentially interacting CMEs, ejected between 2012 June 12-14, that caused a moderate geomagnetic storm with a minimum Dst of -71 nT on 2012 
June 17 14:00 UT.

Figure 1 depicts the planetary and spacecraft positions on 2012 June 14 with Venus located $6^{\circ}$ away from the Sun-Earth line, allowing $V E X$ data to be used as an in situ reference point close to the Sun-Earth line. The two coronagraphs COR2 (part of the SECCHI instrument suite, Howard et al. 2008) on board the two Solar TErestrial RElations Observatory (STEREO, Kaiser et al. 2008) spacecraft provide a stereoscopic side view on the Sun-Earth line and are used to derive CME initial parameters.

In the following sections, we first provide a step-by-step introduction to our method for forecasting Dst. We then discuss observations and provide details of three CMEs that preceded the June 17 geomagnetic storm and may have interacted on their way from the Sun to $V E X$ and Earth. We also discuss further improvements and applications of our method. 


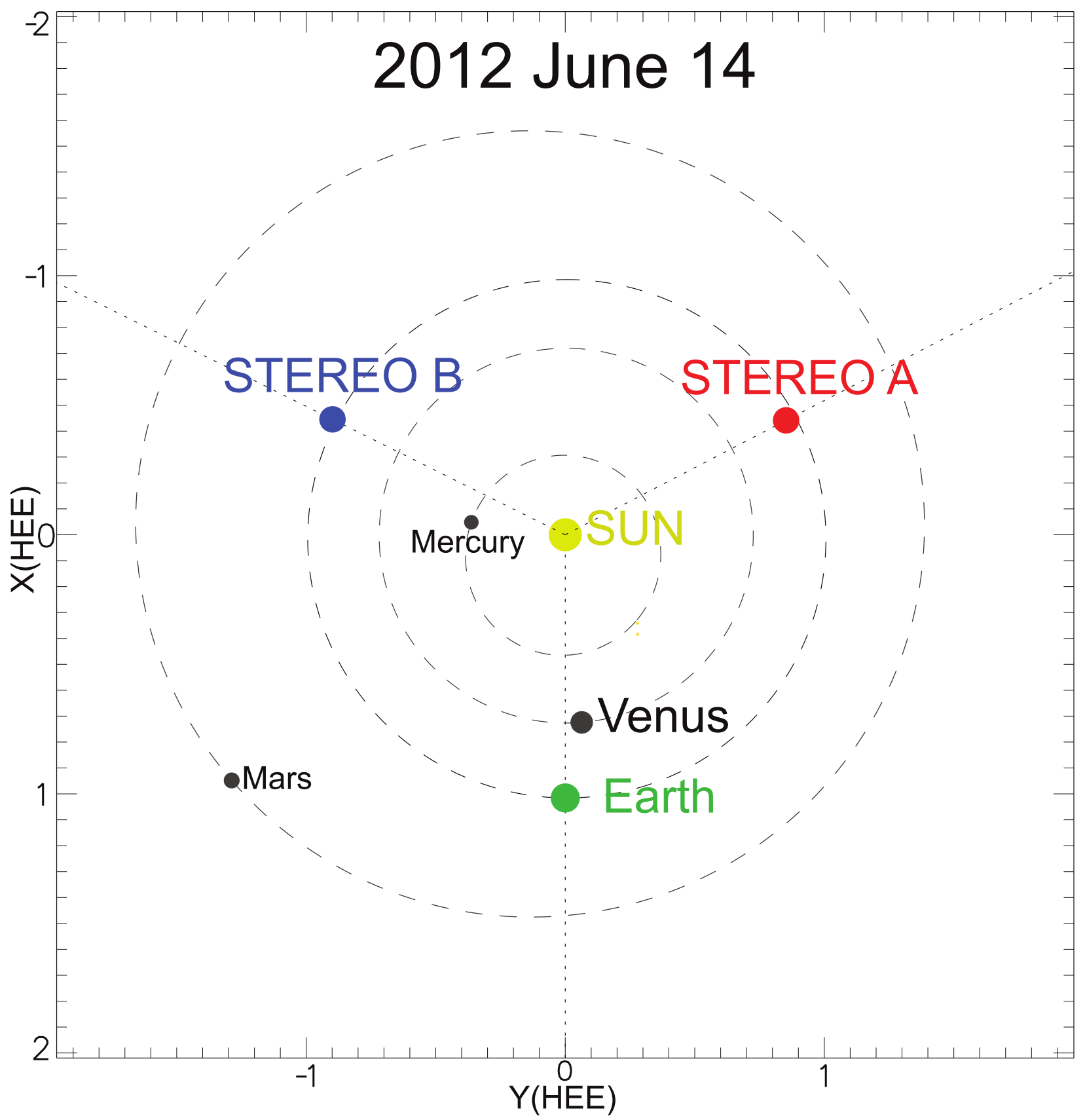

Fig. 1.- Overview of the planetary and spacecraft alignment for the 2012 June 14 CME event. The two STEREO spacecraft provide a side view of the CME along the Sun-Earth line. Venus is located $6^{\circ}$ west of the Sun-Earth line at $0.72 \mathrm{AU}$. 


\section{Method}

We now provide a walkthrough for the necessary steps, parameters and assumptions of our method, starting with the eruption of the CME from the Sun and its evolution through the heliosphere, passing the in situ observing spacecraft and finally leading to geomagnetic activity at Earth. Referring to the constellation Sun - VEX - Earth L1 (see Figure 1), our method can be summarized in the following steps:

1. The CME eruption is imaged with coronagraphs, which yields the CME initial speed and direction.

2. The CME propagation is modeled from Sun to VEX and Wind with the drag-based model (DBM, Vršnak et al. 2013). Inputs are the CME initial speed and the solar wind background speed from a WSA-Enlil simulation run (Arge \& Pizzo 2000; Odstrcil et al. 2004). The output is the CME speed and distance as function of time.

3. The CME propagation with DBM is constrained with the shock arrival time at $V E X$. The input is the time of the CME shock arrival at $V E X$, and the DBM drag-parameter $\gamma$ forms the output of this procedure.

4. The CME magnetic field components observed by $V E X$ are scaled to Earth L1 in magnitude by using an inverse power law in heliocentric distance derived from previous studies. Then the field is shifted in time according to the kinematics obtained from the previous steps.

5. Finally, using the calculated magnetic field and speed of the CME at Earth L1, the time profile of the Dst index is produced.

We now discuss these steps in a more detailed manner before applying them to actual data in Section 3 ,

\subsection{CME kinematics}

We use the DBM from Vršnak et al. (2013) to get the time dependent CME position and speed. Strictly speaking, all arrival times and speeds we quote from DBM are valid for the location of the shock at the front of the ICME. The DBM requires three input parameters, which are: the initial speed of the CME, $v_{0}$, at initial distance from the Sun, $r_{0}$, the solar wind background speed, $w$, and the drag parameter, $\gamma$, so that the shock speed, $v(t)$, and distance from the Sun, $r(t)$, as functions of time are given by: 


$$
\begin{aligned}
v(t) & =\frac{v_{0}-w}{1 \pm \gamma\left(v_{0}-w\right) t}+w \\
r(t) & = \pm \frac{1}{\gamma} \ln \left[1 \pm \gamma\left(v_{0}-w\right) t\right]+w t+r_{0}
\end{aligned}
$$

The \pm solution indicates deceleration or acceleration of the CME by the ambient solar wind, where a plus means deceleration $\left(v_{0}>w\right)$ and minus stands for acceleration $\left(v_{0}<w\right)$.

The CME initial speed is derived from coronagraph observations and for the solar wind background speed $w$ we rely on the WSA-Enlil model because, unfortunately, VEX does not provide plasma observations. The background speed is determined by cutting out a solar wind speed profile from WSA-Enlil along the Sun-Earth line from the start of the CME until its shock arrives at the in situ spacecraft. Obtaining $w$ with the help of WSAEnlil provides a way to avoid using a fixed standard solar wind speed and allows high speed streams to be taken into account. Additionally, this allows an estimate of the speed along the Sun-Earth line that the CME encounters during its propagation from the Sun to Earth, while observations from $V E X$, if available, would only provide the speed at $V E X$.

The drag-parameter, $\gamma$, is a free parameter within a typical range of $0.2 \times 10^{-7}$ to $2 \times 10^{-7} \mathrm{~km}^{-1}$ (Vršnak et al. 2013), although other studies revealed that smaller values of $\gamma$ on the order of $0.01 \times 10^{-7} \mathrm{~km}^{-1}$ are also possible (Rollett et al. 2014; Temmer \& Nitta 2015; Rollett et al. 2016). Knowledge of the shock arrival time at $V E X$ allows us to constrain $\gamma$ in a way that the predicted shock arrival from the DBM at $V E X$ matches the actual, measured arrival time of the shock. This is an important new step, that distinguishes this method from other CME forecasting methods, since once $\gamma$ is fixed, the predicted arrival time and the speed of the shock at Earth L1 can be narrowed down considerably. Using in situ arrival times to constrain CME parameters to study their kinematics has already been introduced by Rollett et al. (2012, 2013, 2014), but here we apply this concept for the first time in order to predict the CME parameters at another location.

There are some limitations in the use of the DBM with a constant $\gamma$ and solar wind speed $w$ regarding the flexibility to adapt to changes in the ambient medium, for example changes in the solar wind speed or to CME-CME interactions, such as in the event discussed in this paper. For future studies the enhanced DBM, which includes a variability for both $\gamma$ and $w$ as introduced by Žic et al. (2015) may also be used for describing the CME kinematics within the framework of our method. 


\subsection{Dst prediction}

To forecast the Dst index from the predicted CME magnetic field and its speed, we use the two different empirical models (Burton et al. 1975, BU75) and O'Brien \& McPherron (2000, OB00). Both models require the ICME dynamic pressure, speed and, most important, the magnetic $B_{z}$ component as input. We can derive the ICME speed from $V E X$ observations (see previous Section) and the derivation of $B_{z}$ will be described in the following paragraphs.

As the CME moves through the heliosphere, its magnetic field strength decreases with increasing distance from the Sun according to a power law, determined from various in situ observations up to 1 AU and beyond (e.g. Bothmer \& Schwenn 1998; Wang et al. 2005; Gulisano et al. 2012; Winslow et al. 2015). A relation for the magnetic field strength of an ICME at the heliocentric distance between $V E X\left(r_{\mathrm{v}}\right)$ at $0.72 \mathrm{AU}$ and Wind $\left(r_{\mathrm{w}}\right)$ at $0.99 \mathrm{AU}$ was derived by Möstl et al. (2012) using the power law from Leitner et al. (2007), based on a set of ICME maximum magnetic fields:

$$
B\left(r_{\mathrm{w}}\right)=B\left(r_{\mathrm{v}}\right)\left(\frac{r_{\mathrm{w}}}{r_{\mathrm{v}}}\right)^{(-1.64 \pm 0.4),}
$$

with $B\left(r_{v}\right)$ and $B\left(r_{w}\right)$ the magnetic field strength at the position of $V E X$ and Wind, respectively. Applying Equation (3) to each of the measured VEX magnetic field components and the total magnetic field results in an appropriately scaled magnetic field strength at the heliocentric distances of Earth L1. If a CME may become deflected in interplanetary space, which means that it would propagate non-radially away from the Sun, the spacecraft located at Earth L1 could pass through different parts of the CME compared to VEX, and the nearEarth spacecraft would measure a deviating magnetic field configuration. However, there is some consensus that the propagation direction of CMEs is largely determined close to the Sun (e.g. Kay \& Opher 2015; Möstl et al. 2015), so we assume here that no interplanetary deflection is taking place. We are aware that this simple assumption is a limiting case for the accuracy of the method but it enables us to study the potential effects of a deflection of the CME that might happen between the two in situ spacecraft.

We also need to take into account the time it takes for the CME to travel from VEX to Earth. This requires the results on CME kinematics by the DBM. We shift the VEX in situ measurements in time by taking the time difference between the measured shock arrival time at $V E X$ and the predicted arrival time from the DBM at the heliocentric distance of Wind.

The last required input parameter for the BU75 and OB00 models is the dynamic 
pressure. For this parameter, $p_{\text {dyn }}$, we assume a typical and constant ICME density of 10 protons $\mathrm{cm}^{-3}$ (Richardson \& Cane 2010). Note that this assumption can lead to an underestimate of $p_{\text {dyn }}$ during the ICME sheath region, which often has a higher density, resulting in a first positive peak in Dst. However, during the flux rope interval in the ICME the density is low (e.g. Richardson \& Cane 2010). Thus, the negative excursion in Dst, which is the main phase of the geomagnetic storm that we are mainly interested in predicting, is not expected to be significantly affected by our assumption of constant and low density.

\section{Data}

\subsection{Imaging observations}

Figure 2 shows two coronagraph images by the COR2 instrument (Howard et al. 2008) on STEREO-A/B, about 1 hour after a major Earth-directed CME first appeared on 2012 June 14 14:09 UT. However, between 2012 June 12 and 14, there were three potentially Earth directed CMEs, one on each day: CME1 on June 12, CME2 on June 13, CME3 on June 14. In order to understand potential effects of each CME on the subsequently measured in situ data at $V E X$ and Wind, we quote results on interplanetary directions and speeds of these 3 CMEs that were obtained with geometrical modeling based on observations by the STEREO Heliospheric Imagers (HI, Evles et al. 2009). We quote values derived from the Fixed $-\Phi$ fitting (FPF, Sheeley et al. 1999; Rouillard et al. 2008), self-similar expansion fitting (SSEF, Davies et al. 2012; Möstl \& Davies 2013, with $30^{\circ}$ half-width) and harmonic mean fitting (HMF, Lugaz et al. 2010; Möstl et al. 2011) techniques. For a further explanation of these methods see Möstl et al. (2014). The following results are taken from the HELCATS webpage1, a project where catalogs of CMEs are established during the STEREO era since April 2007. We quote CME directions in longitude and latitude in the Heliocentric Earth Equatorial (HEEQ) system which are converted from fits to observed time elongationprofiles of the CME leading edges at position angles around the center of the CME (e.g. Rouillard et al. 2009; Möstl et al. 2014).

CME12: In STEREO-A/B/COR2 the event appears first on 2012 June 12 18:54 UT, and in $S O H O / L A S C O / C 3$ the event is a partial halo but very faint. Geometrical modeling based on $S T E R E O-A(B)$ yields a direction ranging from $2^{\circ}$ to $26^{\circ}\left(-26^{\circ}\right.$ to $\left.0^{\circ}\right)$ in $\mathrm{HEEQ}$ longitude and from $-5^{\circ}$ to $-7^{\circ}\left(10^{\circ}\right.$ to $\left.12^{\circ}\right)$ in HEEQ latitude, and a speed from 411 to 453

\footnotetext{
${ }^{1}$ http://www.helcats-fp7.eu/catalogues/wp3_cat.html, accessed on 2016 July 14.

${ }^{2}$ HELCATS catalog id: HCME_A__20120612_02, HCME_B__20120612_01
} 


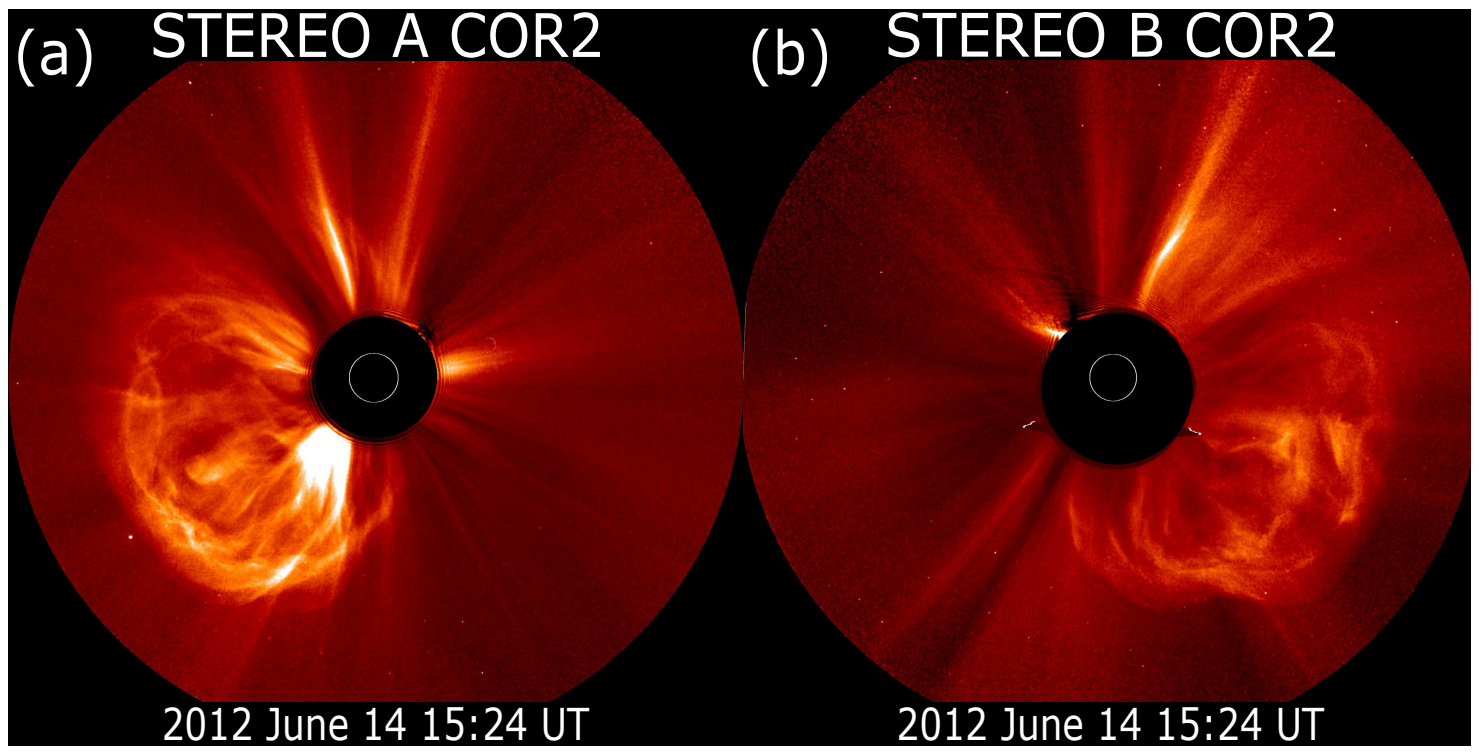

Fig. 2.- The Earth-directed CME3 on 2012 June 14 observed by the STEREO-A (left) and STEREO-B (right) COR2 coronagraphs at 15:24 UT.

$\mathrm{km} \mathrm{s}^{-1}$ (440 to $499 \mathrm{~km} \mathrm{~s}^{-1}$ ). Thus, CME1 is an Earth directed CME, with quite consistent results derived from both $S T E R E O$ viewpoints, with a slow interplanetary propagation speed of approximately $450 \mathrm{~km} \mathrm{~s}^{-1}$.

CME23: In STEREO-A/B/COR2 the event appears first around 2012 June 13 14:00 UT. The direction of CME2 ranges from $33^{\circ}$ to $38^{\circ}\left(-55^{\circ}\right.$ to $\left.-39^{\circ}\right)$ in HEEQ longitude and from $0^{\circ}$ to $1^{\circ}\left(-25^{\circ}\right)$ in HEEQ latitude. Note that the quoted latitude from STEREO$A$ arises from a tracking at a position angle close to the solar equatorial plane, but in all three coronagraphs (STEREO-A/B/COR2 and SOHO/LASCO/C3), this slow CME event is strongly southward directed. The speed ranges from 496 to $500 \mathrm{~km} \mathrm{~s}^{-1}$ (544 to $574 \mathrm{~km} \mathrm{~s}^{-1}$ ). It is striking that the longitudinal direction is inconsistent comparing the results from the two viewpoints of $S T E R E O-A / B$, with a difference of about $80^{\circ}$. In all three coronagraphs the CME also appears asymmetric, which together with the large differences in longitude, may point to a double eruption where neither of the two CMEs is fully earthward directed.

CME34: The direction ranges from $-6^{\circ}$ to $29^{\circ}\left(-36^{\circ}\right.$ to $\left.-7^{\circ}\right)$ in HEEQ longitude and from $-6^{\circ}$ to $-4^{\circ}\left(-1^{\circ}\right.$ to $\left.2^{\circ}\right)$ in latitude with a speed from 791 to $983 \mathrm{~km} \mathrm{~s}^{-1}$ (877 to 1017 $\mathrm{km} \mathrm{s}^{-1}$ ). This is a clearly Earth directed (see Figure 2) and fast CME, with an interplanetary

\footnotetext{
${ }^{3}$ HELCATS catalog id: HCME_A__20120613_01, HCME_B__20120613_01

${ }^{4}$ HELCATS catalog id: HCME_A__20120614_01, HCME_B__20120614_01
} 
propagation speed of about $900 \mathrm{~km} \mathrm{~s}^{-1}$. It is unambiguously related to a M1.9 flare at S19E06 peaking at 14:35 UT, close in time to the first image of the CME in COR2A at 14:09 UT. It is accompanied by two dimming regions and a bright post eruption arcade which are coronal signatures interpreted as caused by an erupting flux rope (e.g. Webb et al. 2000).

In summary, both CME1 and CME3 can be expected to contribute to in situ signatures measured near the Sun - Earth line, and a glancing blow at that location is possible from CME2, due to its mainly southward direction. In order to proceed with calculating the CME kinematics we now need to make an association between an arrival time in the in situ data and the initial speed of one of those 3 CMEs.

\subsection{In situ observations}

Figure 3 shows the in situ magnetic field measurements of VEX and Wind. Since CME3 is Earth directed and has the fastest initial speed of the three CMEs in question, we associate the time where the total field strength makes the most significant jump in the VEX data as being the shock of CME3 (appropriately named S3v), arriving on 2012 June 16 04:54 UT (vertical solid line in Figure $3 \mathrm{a}$ ). This association is also based on the observational fact that faster CMEs have a higher in situ magnetic field strength (Yurchyshyn et al. 2005; Möstl et al. 2014). This means that the high total field following S3v suggests this structure is caused by CME3. Note that for 3 interacting CMEs this association is very difficult to determine unambiguously, and our association of CME3 with S3v could be challenged by exchanging it with the times of the two other shocks present before S3v. Future studies using our new method will thus need to look in further details concerning this association problem and how it affects the prediction of $D s t$.

Figure 3 a also shows that two different flux ropes (two differently shaded areas before and after S3v) immediately follow each other. Magnetic flux ropes are easily discerned in in situ data as they show prolonged, large scale field rotations accompanied by a higher total magnetic field as compared to the background wind (e.g. Burlaga et al. 1981). This suggests that at least two of the CMEs merged before arriving at VEX, assuming that each CME included a flux rope. The presence of at least two more shocks in the VEX data ahead of S3v possibly generated by CME1 and CME2 is also consistent with merging. The black line in Figure 3 $3 \mathrm{~b}$ indicates the most likely arrival of shock S3 at the Wind spacecraft and is appropriately named S3w, arriving on 2012 June 16 19:34 UT, 14 hours and 40 minutes after arriving at $V E X$. In this study, we do not examine further how the various structures observed in situ at VEX and Wind might be related to the three CMEs. Rather we focus on whether the $V E X$ in situ data mapped to Earth can provide a successful prediction of 
the $D s t$ index.
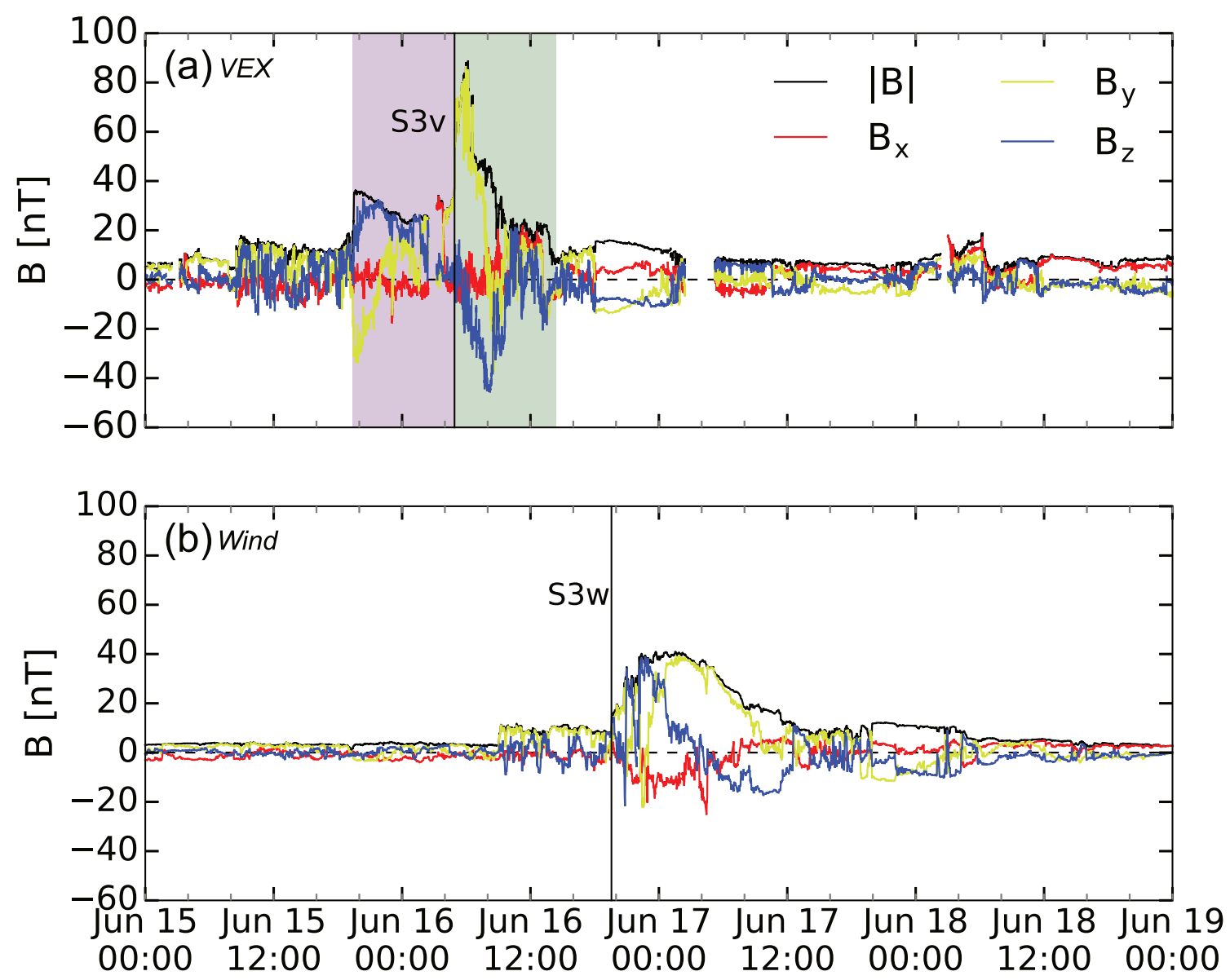

Fig. 3.- In situ magnetic field observations by VEX (VSO coordinates) and Wind (GSE coordinates). (a) Interplanetary magnetic field (IMF) components and total magnetic field at $V E X$. The feature tentatively identified as the shock of CME3, S3v, arriving at VEX on 2012 June 16 04:54 UT is marked as a vertical black line. Evidence for CME-CME interaction as suggested by the two flux ropes (shaded areas) that immediately follow each other. (b) Wind observations of the IMF. The feature identified as the shock S3w arrives at Wind on 2012 June 16 19:34 UT. 


\section{Results}

Figure 4 shows the DBM results for $r(t)$ (the heliocentric Distance in AU) and $v(t)$ using an initial speed of CME3 of $v_{0}=1102 \mathrm{~km} \mathrm{~s}^{-1}$, which was obtained by Möstl et al. (2014) using the graduated cylindrical shell model (Thernisien et al. 2009) at $r_{0}=15$ solar radii, assuming this speed to be valid for the initial shock speed. We used this speed in favor of the speed mentioned in the data Section because of its higher accuracy. This speed is slightly $\left(\approx+150 \mathrm{~km} \mathrm{~s}^{-1}\right)$ higher than the average interplanetary propagation speed from HI modeling. A range of $v_{0}$ from 1000 to $1200 \mathrm{~km} \mathrm{~s}^{-1}$ is taken into account (see black and green lines in the early speed profile in Figure 4b) to include typical uncertainties of speed determination (e.g. Möstl et al. 2014). We have determined the background wind speed by taking an average along the Sun-Earth line in a WSA-Enlil run from the launch of the CME on 2012 June 14 14:09 until the time of the S3v arrival at VEX, resulting in $w=389 \pm 40$ $\mathrm{km} \mathrm{s}^{-1}$. With the arrival time of the shock S3v at VEX (2012 June 16 04:54), $\gamma$ is constrained to the value of $0.271 \times 10^{-7} \mathrm{~km}^{-1}$ for $v_{0}=1102 \mathrm{~km} \mathrm{~s}^{-1}$ (Figure $4 \mathrm{a}$ ). An extrapolation of these kinematics to $0.99 \mathrm{AU}$ in Figure 4a leads to an arrival time at Wind on 2012 June 17 01:42. Setting $v_{0}$ to $1000 \mathrm{~km} \mathrm{~s}^{-1}$ and constraining the fit by the VEX arrival time gives a $\gamma$ of $0.215 \times 10^{-7} \mathrm{~km}^{-1}$ and a shock arrival time at Wind of 2012 June 17 00:52. Respectively, for a $v_{0}$ of $1200 \mathrm{~km} \mathrm{~s}^{-1}$ we obtain a $\gamma$ of $0.315 \times 10^{-7} \mathrm{~km}^{-1}$ and a shock arrival on 2012 June 17 02:16. This shows, that changing the initial speed from $1000 \mathrm{~km} \mathrm{~s}^{-1}$ to $1200 \mathrm{~km} \mathrm{~s}^{-1}$ only changes the Wind arrival time by 84 minutes in total.

The DBM yields an arrival speed at Wind of $531 \mathrm{~km} \mathrm{~s}^{-1}$ and an interesting consequence of constraining the DBM fits using the shock arrival time at VEX is that increasing the initial CME speed counter-intuitively moves the arrival time at Wind later, and also reduces the arrival speed, for example from $554 \mathrm{~km} \mathrm{~s}^{-1}$ for an initial speed of $1000 \mathrm{~km} \mathrm{~s}^{-1}$ to 516 $\mathrm{km} \mathrm{s}^{-1}$ for an initial speed of $1200 \mathrm{~km} \mathrm{~s}^{-1}$. The reason for this is that a larger drag coefficient is required for an initially higher speed CME to match the VEX arrival time, and this greater drag then delays the arrival at Wind and reduces the arrival speed.

Figure 5 a shows the speed profile observed by the Wind SWE instrument (Ogilvie et al. 1995), revealing three major jumps. The first jump early on 2012 June 16, to around 400 $\mathrm{km} \mathrm{s}^{-1}$ from a background wind speed of $300 \mathrm{~km} \mathrm{~s}^{-1}$, most likely stems from one of the previous CMEs. The second jump in speed, labeled S3w, is followed by another jump shortly afterwards. This double-shock feature could stem from two merged CMEs and makes the association of a shock to its corresponding CME somewhat ambiguous, therefore limiting the accuracy in arrival time prediction. Figure 5a also shows the predicted arrival speed (red horizontal line) and its range $\left(516-554 \mathrm{~km} \mathrm{~s}^{-1}\right.$, shaded red area) based on initial CME speeds of $1000-1200 \mathrm{~km} \mathrm{~s}^{-1}$. This is consistent with the observed solar wind speeds 

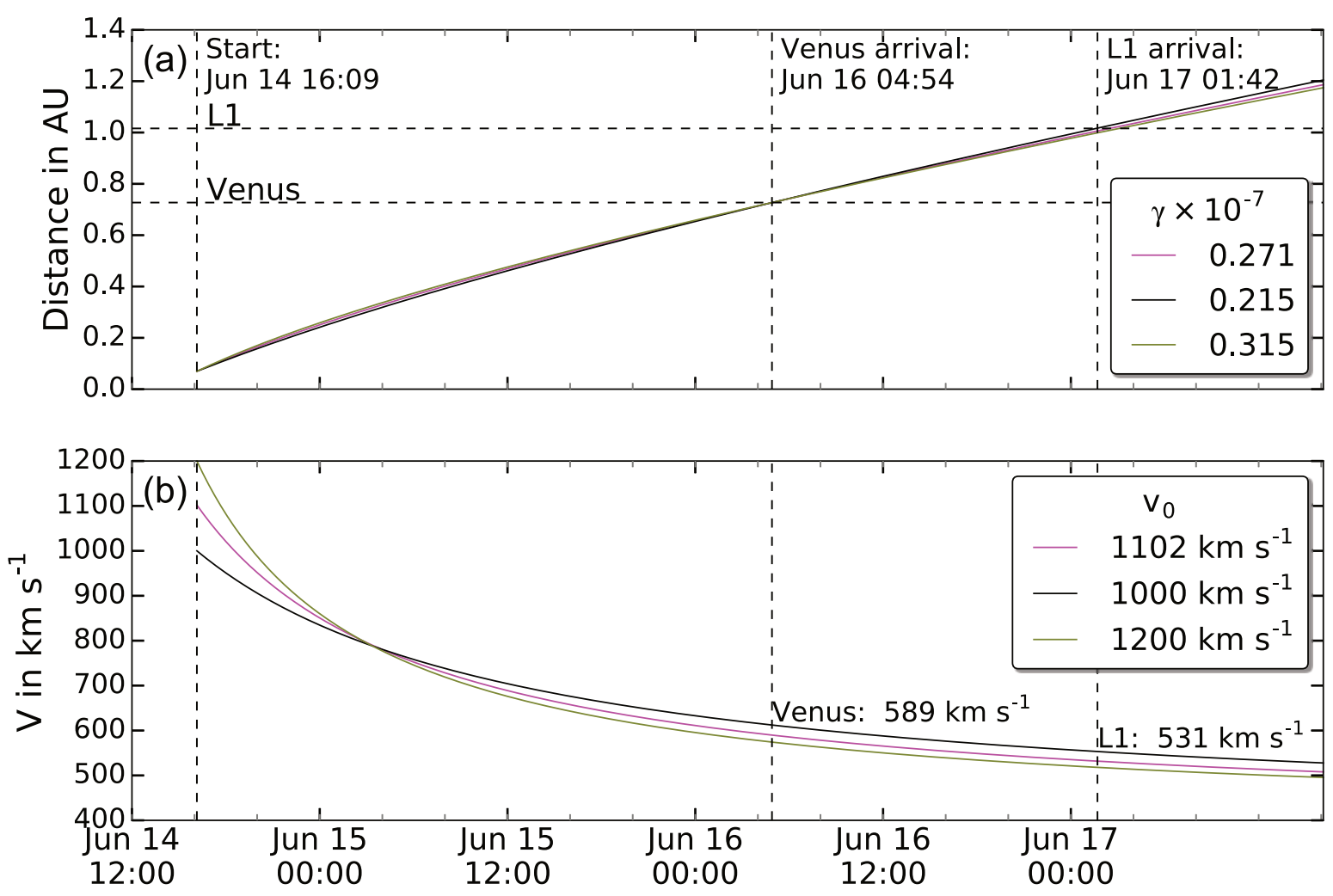

Fig. 4. - CME3 shock kinematics of the 2012 June 14 event, given by the drag-based model (DBM) and constrained with the VEX shock arrival time, S3v. (a) CME3 shock distance versus time. The horizontal dashed lines mark the distances of $V E X$ and L1/Wind. The vertical dashed lines show the start time of CME3 at 15 solar radii, the arrival time at $V E X$ (shock time for S3v) and the predicted arrival at L1/Wind. (b) CME3 shock speed versus time for the initial speed $v_{0}$ of $1102 \mathrm{~km} \mathrm{~s}^{-1}$ (see Section 4). The predicted speeds for VEX and Wind are indicated for $v_{0}=1102 \mathrm{~km} \mathrm{~s}^{-1}$ (magenta). Both panels include kinematics arising from variations of $v_{0}$ to $1200 \mathrm{~km} \mathrm{~s}^{-1}$ (green) and $1000 \mathrm{~km} \mathrm{~s}^{-1}$ (black).

following the third shock. The predicted speed of $531 \pm 23 \mathrm{~km} \mathrm{~s}^{-1}$ (Figure 5a) only deviated by $41 \mathrm{~km} \mathrm{~s}^{-1}$ from the measured value of $490 \pm 30 \mathrm{~km} \mathrm{~s}^{-1}$, for which we took an average speed during the sheath region, from S3w to June 17 00:00 UT.

For calculating the Dst index, the ICME speed was set to $531 \pm 23 \mathrm{~km} \mathrm{~s}^{-1}$ (including the variations of $v_{0}$ as described above) throughout the ICME and $B_{z}$ is taken from the scaled and time shifted VEX observations, done according to Section 2.2. We shift the 
$V E X$ in situ measurements in time by taking the time difference of 20 hours and 46 minutes between predicted arrival time from the DBM at L1 (June 17 01:40) and the measured shock arrival time of CME3 (S3v) at VEX (June 16 04:54). This time of about 21 hours is also the lead time of the prediction. Figure 50 shows a prediction of the magnetic field corrected for both strength and timing at Earth L1 by applying our method to measurements from VEX at 0.72 AU. Figure 5b shows the magnetometer data from Wind for a direct comparison, demonstrating a decent though certainly not perfect match in timing, field strength and the behavior of $B_{z}$.

Figure 5 5 shows the prediction of the Dst index with our method, solely based on scaled data from $0.72 \mathrm{AU}$, in comparison to the actual Dst (black dots, provided by the World Data Center for Geomagnetism, Kyoto). The blue line is the Dst prediction using the BU75 model and red shows the OB00 result. Shaded areas mark vertical errors in Dst, which arise (1) from the uncertainty in the exponent of the power law in Equation (3) and (2) from a variation of the predicted CME arrival speed at Earth L1. The horizontal error bars in Figure $5 \mathrm{~d}$ indicate errors in the predicted arrival time due to uncertainties in the ICMEs initial speed $v_{0} \pm 100 \mathrm{~km} \mathrm{~s}^{-1}$. For better visibility, these horizontal bars are only drawn for the period of decreasing Dst. The measured Dst shows a minimum of $-71 \mathrm{nT}$ and the BU75 (OB00) models yield a minimum Dst of $-96(-114) \mathrm{nT}$ with an upper limit of $-83(-96) \mathrm{nT}$ and a lower limit of $-113(-141) \mathrm{nT}$. The average predicted values minimum $D$ st values are -25 $\mathrm{nT}(\mathrm{BU} 75)$ and $-43 \mathrm{nT}(\mathrm{OB} 00)$ below the observed one, which is a rather promising result.

\section{Discussion}

To calculate a prediction for the Dst index out of measurements from $V E X$ at $0.72 \mathrm{AU}$, we started with the DBM to obtain the arrival time and the speed of the CME at L1. The solar wind background speed for the DBM was derived by data from the WSA-Enlil model rather than setting the solar wind speed to a default value. In doing so, we are able to take solar wind high speed streams into account. There is potential for improving the method regarding cases where a CME is traveling through more distorted solar wind.

Note that, counterintuitively, the highest $v_{0}$ results in the latest arrival time, as well as the lowest arrival speed at Wind and vice versa, because $\gamma$ is constrained to the ICME arrival time at the in situ spacecraft (see also Figure 4). Determination of $\gamma$ in this way has the advantage of a good estimate of its average value up to the in situ spacecraft. On the other hand, constraining $\gamma$ at the in situ spacecraft and assuming a constant $\gamma$ all the way from the Sun to Earth may lead to inaccurate results if the in situ spacecraft (the one at $<1 \mathrm{AU}$ ) would be located at a heliocentric distance closer to the Sun, and a variable $\gamma$ 


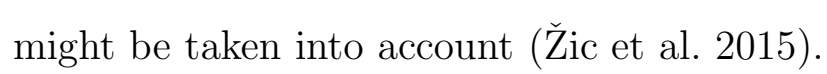

Compared to the true shock arrival at L1 on 2012 June 16 19:34 UT (S3w, Figure 5b) the DBM predicted the shock at 2012 June 17 01:40 UT (S3, Fig. 55), about 6 hours too late, which is clearly above the error of \pm 1 hour associated with this arrival time from our calculations. As an explanation for the discrepancy between predicted and actual arrival time of 6 hours we suggest two hypothesis: (1) A local distortion of the shock front. (2) The association between the measured magnetic field structures that arrive at VEX and Wind is ambiguous for this event and might be incorrect.

For point (1), consider the following: $V E X$ is located about $6^{\circ}$ away from the Sun-Earth line in heliospheric longitude which is equivalent to $0.075 \mathrm{AU}$ (at VEX distance) and 0.105 AU (at Earth distance). An interaction of CME3 with one or both of the two preceding CMEs might have distorted the CME shock shape in such a way that the part of shock S3 that travels along the Sun-Earth line has already traveled further in heliocentric distance than the part that impacted $V E X$. This could be explained by an inclination of the shock surface. However, if we assume that the shock traveled for 6 hours with a speed of 530 $\mathrm{km} \mathrm{s}^{-1}$, the resulting radial distance between the the part of the shock that hits $V E X$ and the part that travels along the Sun-Earth line would be in the order of $0.08 \mathrm{AU}$, thus the possible inclination of the shock surface would be very significant. If hypothesis (2) holds and the magnetic field structures at $V E X$ and Wind are incorrectly associated, the error between predicted and measured arrival could be different, but this "association problem" will be further discussed in future studies.

Aside these two hypotheses, we can rule out one other potential factor that could be responsible for the arrival time discrepancy, namely an incorrect determination of the DBM drag parameter $\gamma$. In order to cover the distance of 0.28 AU from VEX to Earth L1 within the time interval 2012 June 16 04:54 (S3 at VEX) and June 16 19:34 (S3w at Wind) it would require an average speed of $\sim 790 \mathrm{~km} \mathrm{~s}^{-1}$. This means that the speed at $V E X$ would even be way higher, leading to an unlikely scenario where virtually no deceleration of the shock takes place from the Sun to $\operatorname{VEX}($ low $\gamma$ ) and all the deceleration happens between $V E X$ and Earth (very high $\gamma$ ). Such a scenario might be possible if CME3 had interacted with one of the preceding CMEs somewhere between $V E X$ and Earth, but $V E X$ data shows that the interaction already occurred before CME3 hit $V E X$ (see Figure $3 \mathrm{a}$ ).

To predict the magnetic field at Earth L1, we have applied Equation (3) to each magnetic field component from VEX measurements individually, although previous work on scaling of the magnetic field inside flux ropes suggest that the axial and azimuthal components may scale with $1 / r^{2}$ and $1 / r$ respectively (e.g. Osherovich et al. 1993; Farrugia et al. 1993). However, the exponent $-1.64 \pm 0.4$ covers most of this range and a different scaling law may 
be added in future studies.

\section{Conclusions}

We showed that the problem of predicting the geomagnetic effects of CMEs may in principle be solved by using in situ magnetometer observations by a spacecraft located in the inner heliosphere close to the Sun-Earth line, thereby strongly extending the method of Lindsay et al. (1999). The position of the in situ spacecraft is a trade-off between prediction accuracy and lead time, where a spacecraft close to Earth provides high accuracy forecasts at the cost of short lead time. We have studied a CME which led to a moderate geomagnetic storm at Earth and likely interacted with two preceding CMEs. We applied for the first time a new method consisting of several steps to predict the Dst time profile. With data from $V E X$, located at 0.72 , AU we obtained a relatively accurate $D$ st forecast ranging from of -83 to $-141 \mathrm{nT}$ (including error bars), near the observed Dst of $-71 \mathrm{nT}$, while still maintaining a prediction lead time of nearly 21 hours. Knowing the time of CME ejection at the Sun on 2012 June 14 14:09 UT and the arrival at $V E X$ on 2012 June 16 allows us to tightly constrain the CME kinematics, leading to a predicted CME arrival speed at Earth of $531 \pm 23 \mathrm{~km} \mathrm{~s}^{-1}$, similar to the observed $490 \pm 30 \mathrm{~km} \mathrm{~s}^{-1}$ at Wind.

The predicted arrival time of the CME at Earth is 2012 June 17 01:40 UT, which is 6 1 hours later than the true shock arrival. This discrepancy in arrival time might be explained by an incorrect association between the measured magnetic field structures at $V E X$ and Wind or by an inclination of the shock surface on a scale of $0.08 \mathrm{AU}$ in radial distance. If the shock surface indeed shows such a pronounced local feature, this means that there may exist a limit for the errors in arrival time prediction of CMEs on the order of 6 hours, which cannot be further improved unless the shock shape is modeled very accurately on scales of $<0.1$ AU. Our method thus also provides a new tool for detailed diagnostics on CME physics that allows to reveal those small-scale structures.

Our obtained results for Dst are relatively accurate despite the interaction of several CMEs before they impacted $V E X$ and despite the assumptions that were made regarding the solar wind background speed estimation, magnetic field scaling and determination of the drag parameter $\gamma$. To further improve the accuracy of the results, a better solar wind background speed estimation can be made by a plasma instrument on the in situ spacecraft. This would allow to map a speed profile that includes any variations in the solar wind background speed, and it would allow an even more constrained calculation of the CME kinematics.

The DBM we used is designed for constant input parameters, so further studies on 
more events and different heliospheric distances of the in situ spacecraft will reveal if the assumption of a constant $\gamma$ and $w$ is sufficient, or if the enhanced DBM (Žic et al. 2015) with variable $\gamma$ and $w$ may provide better results.

Other improvements of our method include modeling of the expansion of the CME flux rope (e.g. Démoulin \& Dasso 2009), even with different power laws for the azimuthal and axial fields (e.g. Osherovich et al. 1993), a conversion of the predicted $B_{z}$ component to GSM coordinates, including the CME shock shape (Möstl \& Davies 2013; Möstl et al. 2015), adding a variable dynamic pressure profile, and using an updated method for predicting Dst from solar wind parameters (Temerin \& Li 2006). Because we have assumed a constant ICME density, the positive peak in $D s t$ in Figure $5 \mathrm{~d}$, which stems from a very high density and thus dynamic pressure, is not included in the Dst forecast. However, this peak maximizes at $+95 \mathrm{nT}$ late on 2012 June 16, which is actually the highest positive Dst value in the entire space age, at least since 1957. The impact of these CMEs on Earth has resulted in a strong magnetospheric compression, and a study of the exact interplanetary causes would be worthwhile.

An application of our method to real time magnetic field data by possible future spacecraft thus provides the possibility to make appropriate and timely arrangements to prepare for the potential effects of CMEs on the technological infrastructure of mankind. Our proofof-concept study may be extended, using any spacecraft temporarily located close to the Sun-Earth line (see Lindsay et al. 1999), such as MESSENGER in conjunction with an L1 spacecraft. In addition any line-up of two spacecraft like VEX - STEREO or MESSENGER - STEREO can be used to predict CME arrival times and speeds, as well as magnetic fields from the inner to the outer spacecraft (e.g. Good et al. 2015; Winslow et al. 2016). It can be applied to future space missions in the inner heliosphere like BepiColombo, Solar Probe Plus or Solar Orbiter, or to data from permanent in situ observers at an artificial Lagrange point like the Sunjammer concept (Eastwood et al. 2015), which would allow a continuous, gap-free forecast of the Dst index. It may also be applied to data by a future ring of spacecraft around the Sun (Ritter et al. 2015), carrying at least a magnetometer, where each spacecraft would drift towards and away from the Sun-Earth line. Its feasibility and number of spacecraft needed depends mainly on the longitudinal extent of CME flux ropes (e.g. Bothmer \& Schwenn 1998; Good \& Forsyth 2016), which also warrants further study.

M.K, C.M. and T.A. thank the Austrian Science Fund (FWF): [P26174-N27]. The presented work has received funding from the European Union Seventh Framework Programme (FP7/2007-2013) under grant agreement no. 606692 [HELCATS]. 


\section{REFERENCES}

Arge, C. N., \& Pizzo, V. J. 2000, J. Geophys. Res., 105, 10465

Bala, R., \& Reiff, P. 2012, Space Weather, 10, S06001

Bothmer, V., \& Schwenn, R. 1998, Annales Geophysicae, 16, 1

Burlaga, L., Sittler, E., Mariani, F., \& Schwenn, R. 1981, J. Geophys. Res., 86, 6673

Burton, R. K., McPherron, R. L., \& Russell, C. T. 1975, J. Geophys. Res., 80, 4204

Colaninno, R. C., Vourlidas, A., \& Wu, C. C. 2013, Journal of Geophysical Research (Space Physics), 118, 6866

Davies, J. A., et al. 2012, ApJ, 750, 23

Démoulin, P., \& Dasso, S. 2009, A\&A, 498, 551

Dungey, J. W. 1961, Physical Review Letters, 6, 47

Eastwood, J. P., Kataria, D. O., McInnes, C. R., Barnes, N. C., \& Mulligan, P. 2015, Weather, 70, 27

Eyles, C. J., et al. 2009, Sol. Phys., 254, 387

Farrugia, C. J., Burlaga, L. F., Osherovich, V. A., Richardson, I. G., Freeman, M. P., Lepping, R. P., \& Lazarus, A. J. 1993, J. Geophys. Res., 98, 7621

Good, S. W., \& Forsyth, R. J. 2016, Sol. Phys., 291, 239

Good, S. W., Forsyth, R. J., Raines, J. M., Gershman, D. J., Slavin, J. A., \& Zurbuchen, T. H. 2015, ApJ, 807, 177

Gulisano, A. M., Démoulin, P., Dasso, S., \& Rodriguez, L. 2012, A\&A, 543, A107

Hoeksema, J. T., \& Zhao, X. 1992, J. Geophys. Res., 97, 3151

Howard, R. A., et al. 2008, Space Science Reviews, 136, 67

Kaiser, M. L., Kucera, T. A., Davila, J. M., St. Cyr, O. C., Guhathakurta, M., \& Christian, E. 2008, Space Science Reviews, 136, 5

Kay, C., \& Opher, M. 2015, ApJ, 811, L36 
Leitner, M., Farrugia, C. J., Möstl, C., Ogilvie, K. W., Galvin, A. B., Schwenn, R., \& Biernat, H. K. 2007, Journal of Geophysical Research (Space Physics), 112, 6113

Lindsay, G. M., Russell, C. T., \& Luhmann, J. G. 1999, J. Geophys. Res., 104, 10335

Lugaz, N., Hernandez-Charpak, J. N., Roussev, I. I., Davis, C. J., Vourlidas, A., \& Davies, J. A. 2010, ApJ, 715, 493

Mays, M. L., et al. 2015, Sol. Phys., 290, 1775

Möstl, C., \& Davies, J. A. 2013, Sol. Phys., 285, 411

Möstl, C., et al. 2011, ApJ, 741, 34

-. 2012, ApJ, 758, 10

-. 2014, ApJ, 787, 119

—. 2015, Nature Communications, 6, 7135

O'Brien, T. P., \& McPherron, R. L. 2000, Journal of Atmospheric and Solar-Terrestrial Physics, 62, 1295

Odstrcil, D., Riley, P., \& Zhao, X. P. 2004, Journal of Geophysical Research (Space Physics), 109,2116

Ogilvie, K. W., et al. 1995, Space Science Reviews, 71, 55

Osherovich, V. A., Farrugia, C. J., \& Burlaga, L. F. 1993, Advances in Space Research, 13, 57

Richardson, I. G., \& Cane, H. V. 2010, Sol. Phys., 264, 189

Ritter, B., et al. 2015, Journal of Space Weather and Space Climate, 5, A3

Rollett, T., Möstl, C., Isavnin, A., Davies, J. A., Kubicka, M., Amerstorfer, U. V., \& Harrison, R. A. 2016, ApJ, 824, 131

Rollett, T., Möstl, C., Temmer, M., Veronig, A. M., Farrugia, C. J., \& Biernat, H. K. 2012, Sol. Phys., 276, 293

Rollett, T., Temmer, M., Möstl, C., Lugaz, N., Veronig, A. M., \& Möstl, U. V. 2013, Sol. Phys., 283, 541

Rollett, T., et al. 2014, ApJ, 790, L6 
Rostoker, G., \& Fälthammar, C.-G. 1967, J. Geophys. Res., 72, 5853

Rouillard, A. P., et al. 2008, Geophys. Res. Lett., 35, 10110

—. 2009, Sol. Phys., 256, 307

-. 2011, ApJ, 735, 7

Savani, N. P., et al. 2015, Space Weather, 13, 374

Sheeley, N. R., Walters, J. H., Wang, Y., \& Howard, R. A. 1999, J. Geophys. Res., 104, 24739

Temerin, M., \& Li, X. 2006, Journal of Geophysical Research (Space Physics), 111, A04221

Temmer, M., \& Nitta, N. V. 2015, Sol. Phys., 290, 919

Thernisien, A., Vourlidas, A., \& Howard, R. A. 2009, Sol. Phys., 256, 111

Tobiska, W. K., et al. 2013, Space Weather, 11, 490

Žic, T., Vršnak, B., \& Temmer, M. 2015, ApJS, 218, 32

Vršnak, B., et al. 2013, Sol. Phys., 285, 295

-. 2014, ApJS, 213, 21

Wang, C., Du, D., \& Richardson, J. D. 2005, Journal of Geophysical Research (Space Physics), 110, A10107

Webb, D. F., Lepping, R. P., Burlaga, L. F., DeForest, C. E., Larson, D. E., Martin, S. F., Plunkett, S. P., \& Rust, D. M. 2000, J. Geophys. Res., 105, 27251

Winslow, R., et al. 2016, Journal of Geophysical Research (Space Physics), in press

Winslow, R. M., Lugaz, N., Philpott, L. C., Schwadron, N. A., Farrugia, C. J., Anderson, B. J., \& Smith, C. W. 2015, Journal of Geophysical Research (Space Physics), 120, 6101

Yurchyshyn, V., Hu, Q., \& Abramenko, V. 2005, Space Weather, 3, 8

Zhang, J., et al. 2007, Journal of Geophysical Research (Space Physics), 112, 10102 

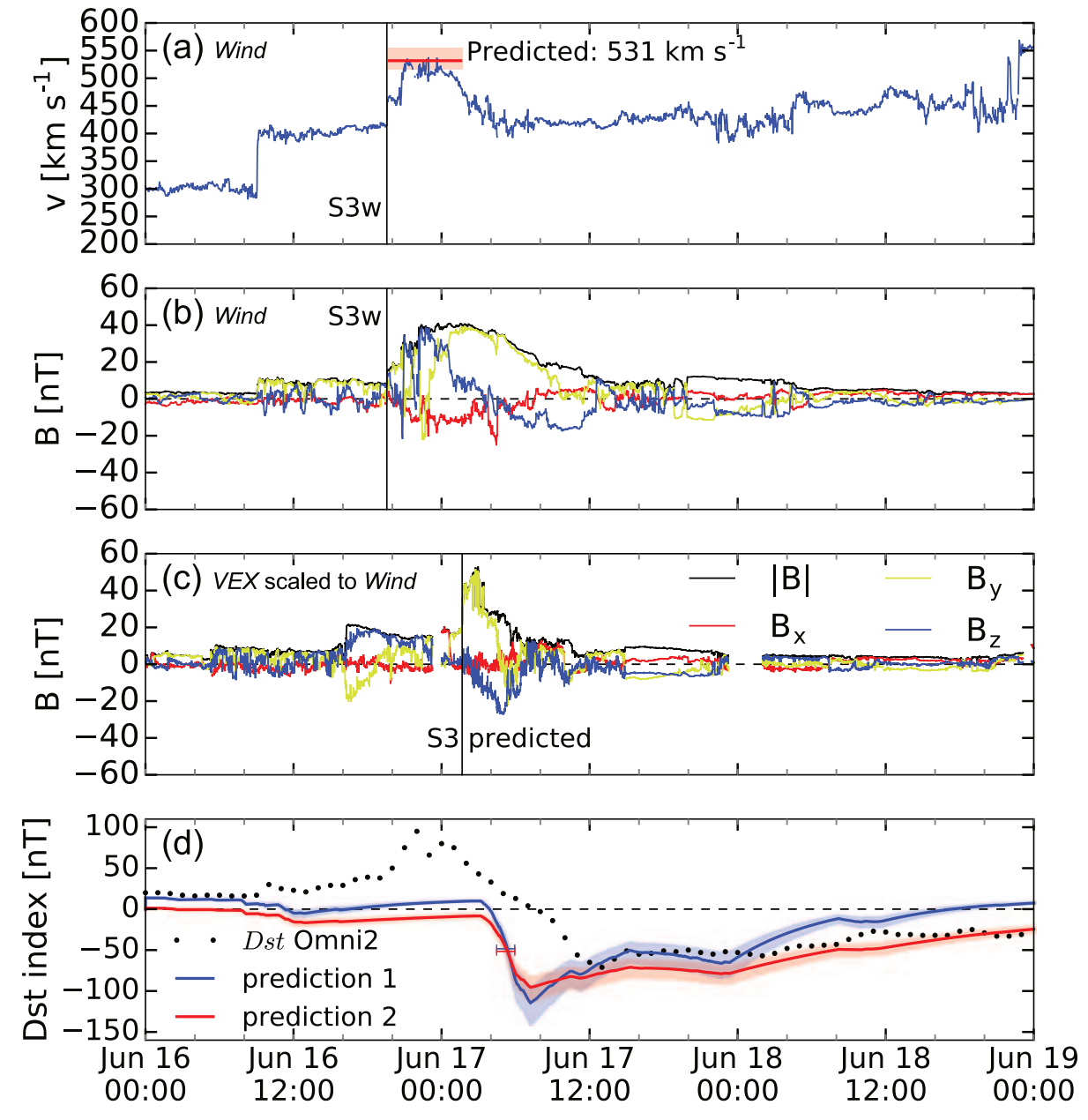

Fig. 5.- Observations by Wind compared to predicted magnetic field components based on VEX data and geomagnetic response. (a) Solar wind speed measured by Wind compared to the predicted shock arrival speed and the range in this speed for initial CME speeds of $1000-1200 \mathrm{~km} \mathrm{~s}^{-1}$, including the arrival time S3w (solid vertical black line). (b) Magnetic field measured by Wind with the feature identified as shock S3w marked as solid vertical black line. (c) IMF scaled from VEX to Wind by a power law (see Section 2.2) and time shifted according to the predicted arrival time of the shock S3 at Wind, which is indicated as solid vertical black line. (d) Predicted Dst using our modeled data as input for Burton et al. (1975) (blue) and O'Brien \& McPherron (2000) (red), compared to the observed Dst (black dots). The shaded areas include errors due to uncertainties in CME initial speed and magnetic field scaling factor (see Sections 2.1 and 2.2). The horizontal error bars indicate the errors in the predicted Dst profiles due to errors in the Earth arrival time prediction. 